\title{
Dataset for Beam Commissioning of the Vero4DRT System
}

\author{
Hideharu Miura1 ${ }^{*}$, Shuichi Ozawa1,2, Shintaro Tsuda1, Masahiro Hayata1, Kiyoshi Yamada1, \\ Yasushi Nagata1,2 \\ ${ }^{1}$ Hiroshima High-Precision Radiotherapy Cancer Center, Hiroshima, Japan \\ ${ }^{2}$ Department of Radiation Oncology, Institute of Biomedical \& Health Science, Hiroshima University, Hiroshima, \\ Japan \\ Email: "miura@hiprac.jp
}

Received 6 November 2015; accepted 20 February 2016; published 23 February 2016

Copyright (C) 2016 by authors and Scientific Research Publishing Inc.

This work is licensed under the Creative Commons Attribution International License (CC BY).

http://creativecommons.org/licenses/by/4.0/

(c) () Open Access

\section{Abstract}

We present the results of measurements made using the Vero4DRT radiation therapy system, which is not yet widely used, to assist technicians in achieving reliable and safe radiotherapy to the patient. We measured percent depth dose, beam profile, and relative scatter factor under water and air conditions. The Vero4DRT system has a $150 \times 150-\mathrm{mm}$ fixed secondary collimator. Its multileaf collimator (MLC) design is a single-focus type, with 30 pairs of $5 \mathrm{~mm}$ thick leaves at the isocenter, and produces a maximum field size of $150 \times 150 \mathrm{~mm}$. Profile measurements were performed using a 0.016- $\mathrm{cm}^{3}$ ionization chamber (PTW31016 pinpoint chamber; PTW, Freiburg GmbH Germany). A brass build-up cap was used for measurements obtained in air conditions. We present a useful measurement dataset for users of the Vero4DRT system.

\section{Keywords}

Vero4DRT, Beam Characteristic, Commissioning

\section{Introduction}

Beamdata commissioning is an important task when delivering radiotherapy doses to patients. The American Association of Physicists in Medicine (AAPM) Task Group 106 reports on phantom and detector setups and measurement techniques to acquire data on beam accuracy [1]. Measurement results may be inaccurate even if beam data are measured according to the reference guide and recommendation files. Even if the sample beam data already may be input into the treatment-planning system (TPS), the vendor may not have provided reliable

${ }^{*}$ Corresponding author.

How to cite this paper: Miura, H., Ozawa, S., Tsuda, S., Hayata, M., Yamada, K. and Nagata, Y. (2016) Dataset for Beam Commissioning of the Vero4DRT System. International Journal of Medical Physics, Clinical Engineering and Radiation Oncology, 5, 70-77. http://dx.doi.org/10.4236/ijmpcero.2016.51007 
data for verifying the user's commissioning results. Because the TrueBeam (Varian Medical Systems, Palo Alto, CA) and NovalisTx devices have been used widely and investigated at many institutions, useful information is available regarding commissioning of this type of linear accelerator [2] [3]. The reliability of measurement results should be enhanced by comparison with these published data.

The Vero4DRT (MHI-TM2000) system was newly developed by Mitsubishi Heavy Industries, Ltd., Japan (MHI) in collaboration with Kyoto University and the Institute of Biomedical Research and Innovation (IBRI). The characteristics of the Vero4DRT system have been described previously [4]-[6]. Briefly, the device is equipped with a $6 \mathrm{MV} \mathrm{X}$-ray beam and an output dose rate of up to $500 \mathrm{MU} / \mathrm{min}$. The Linac head is composed of a compact C-band 6-MV accelerator tube, target, flattening filter, primary collimator, fixed secondary collimator, and multileaf collimator (MLC). The MLC is positioned just below the fixed secondary collimator. The MLC design is a single-focus type, with 30 pairs of $5 \mathrm{~mm}$ thick leaves at the isocenter, and produces a maximum field size of $150 \times 150 \mathrm{~mm}$. Vero4DRT system has a high precision isocenter at the mechanical center of the gantry, which is shaped like an O-ring. The X-ray head with the gimbals can be rotated on the O-ring and moved to pan and tilt directions for dynamic tumor tracking (DTT). The Vero4DRT system is not yet widely in use worldwide (24 Linac devices as of October 2015); thus, no useful information for commissioning is available to compare measurement data. We present our measurement results for percent depth dose (PDD), beam profile, and relative scatter factor in water and air conditions for the Vero4DRT system.

\section{Materials and Methods}

The current Vero4DRT system supports only the iPlan RT dose TPS (BrainLAB, Feldkirchen, Germany), which is available with pencil-beam (PB) and Monte Carlo (MC) algorithms.

\subsection{Measurement Devices}

Measurements were made using the OmniPro-I'mRT software (OmniPro-I'mRT 1.6 IBA dosimetry, Germany) in a Blue Phantom water tank (IBA Dosimetry GmbH, Germany). Profile measurements were performed using a 0.016- $\mathrm{cm}^{3}$ ionization chamber (PTW31016 pinpoint chamber; PTW, Freiburg GmbH, Germany). A brass buildup cap was used for measurements in air to remove electron contamination of the Linac head from the measurement signal. MLC leakage was measured with a Farmer-type ionization chamber (Model N30013; PTW, Freiburg, Germany) with a RAMTEC Smart ${ }^{\mathrm{TM}}$ electrometer (Toyo Medic, Tokyo, Japan).

\subsection{Measurement}

The measurement procedure is described in the "BRAINLAB PHYSICS Technical Reference guide" [7] and are summarized in Tables 1-3. Briefly, the beam dataset for the PB algorithm was acquired on the basis of the following setup conditions: the source surface distance (SSD) was always set at $1000 \mathrm{~mm}$ for profile measurements and the depth was set at $100 \mathrm{~mm}$ for the measurement of relative scatter data. Beam profiles were measured directly under the MLC in such a way that they are not influenced by the interleaf or intraleaf gap. The origin's coordinate is located at the surface of the water phantom.

The beam dataset for the MC algorithm was acquired on the basis of the following setup conditions: the SSD was set at 900 or $1000 \mathrm{~mm}$ for PDD and profile measurements, and the depth was set at $100 \mathrm{~mm}$ for the mea-

Table 1. Dataset for Pencil Beam in water.

\begin{tabular}{|c|c|c|c|}
\hline & Field size (mm) & Depth (mm) & $\mathrm{SSD}(\mathrm{mm})$ \\
\hline PDD & $\begin{array}{c}10 \times 10,20 \times 20,30 \times 30,40 \times 40,60 \times 60 \\
80 \times 80,100 \times 100,120 \times 120,150 \times 150\end{array}$ & $0-350$ & 1000 \\
\hline Relative scatter factor & $\begin{array}{c}10 \times 10,20 \times 20,30 \times 30,40 \times 40,60 \times 60 \\
80 \times 80,100 \times 100,120 \times 120,150 \times 150\end{array}$ & 100 & 1000 \\
\hline Diagonal beam profile & $150 \times 150$ & $5,14,25,50,100,200,350$ & 1000 \\
\hline Transverse beam profile & predefined MLC pattern & dmax, 100, 200 & 1000 \\
\hline MLC leakage & closed MLC closed/opened MLC & 100 & 1000 \\
\hline
\end{tabular}


Table 2. Dataset for Monte Carlo in water.

\begin{tabular}{cccc}
\hline & Field size $(\mathrm{mm})$ & Depth $(\mathrm{mm})$ & SSD $(\mathrm{mm})$ \\
\hline PDD & $100 \times 100$ & $0-350$ & 1000 \\
Transverse beam profile & $100 \times 100$ & dmax, 100, 200 & 1000 \\
PDD & $10 \times 10,30 \times 30,50 \times 50,70 \times 70,100 \times 100$, & & 900 \\
& $150 \times 150,50 \times 150,100 \times 150,150 \times 50,150 \times 100$ & $0-350$ & 900 \\
Transverse beam profile & $10 \times 10,30 \times 30,50 \times 50,70 \times 70,100 \times 100$, & dmax, 100,200 & 900 \\
Relative scatter factor & $150 \times 150,50 \times 150,100 \times 150,150 \times 50,150 \times 100$ & & 100 \\
\hline
\end{tabular}

Table 3. Dataset for Monte Carlo in air.

\begin{tabular}{ccc}
\hline & Field size $(\mathrm{mm})$ & Depth $(\mathrm{mm})$ \\
\hline Cax profile & $20 \times 20,30 \times 30,50 \times 50,70 \times 70,100 \times 100$, & $850-1150$ \\
& $150 \times 150,50 \times 150,100 \times 150,150 \times 50,150 \times 100$ & \\
Transverse beam profile & $20 \times 20,30 \times 30,50 \times 50,70 \times 70,100 \times 100$, & $850,1000,1150$ \\
& $150 \times 150,50 \times 150,100 \times 150,150 \times 50,150 \times 100$ & 1000 \\
Relative scatter factor & $20 \times 20,30 \times 30,50 \times 50,70 \times 70,100 \times 100$, &
\end{tabular}

surement of relative scatter data in water. The MC algorithm requires the measurement to be made in air. For measurements in air, the origin of the coordinate system is not located in the isocenter, but in the target. An ionization chamber with a brass build-up cap was used to measure the profiles in air.

For measurement results in water, PDD were normalized to the depth of $14 \mathrm{~mm}$ (maximum depth) and the cross-plane and in-plane profiles were normalized at the central axis (CAX). Beam profiles were normalized at the CAX. Relative scatter data were calculated by dividing the measured data for each MLC setting by the measured data at the MLC setting of $100 \times 100 \mathrm{~mm}$.

\section{Results}

\subsection{Measurement for the PB Algorithm}

Figure 1 shows the PDD curves with an SSD of $1000 \mathrm{~mm}$. Nine fields were measured and depth ranged from 0 to $350 \mathrm{~mm}$. Figure 2 shows the diagonal beam profiles with SSD of $1000 \mathrm{~mm}$ measured at different depths at $150 \times 150 \mathrm{~mm}^{2}$. For diagonal beam profiles, seven depths were measured and radius ranged from -220 to 220 mm. Figure 3 shows the transverse beam (Figure 3(a)) cross-plane and (Figure 3(b)) in-plane profiles with a predefined MLC pattern (Figure 3(c)) measured at 14 (dmax), 100, and $200 \mathrm{~mm}$ depths at $150 \times 150 \mathrm{~mm}^{2}$. The relative scatter data were measured with various field sizes at the depth of $100 \mathrm{~mm}$ with an SSD of $1000 \mathrm{~mm}$. The relative scatter factors ranged from 0.697 at $10 \times 10 \mathrm{~mm}$ to 1.046 at $150 \times 150 \mathrm{~mm}$ (Table 4). The leakage for closed MLC was $0.14 \%$.

\subsection{Measurement for MC Algorithm in Water}

Figure 4 shows the percent depth dose curves with SSD of $1000 \mathrm{~mm}$ normalized to the depth of $14 \mathrm{~mm}$. The $100 \times 100 \mathrm{~mm}$ fields were measured and depth ranged from 0 to $350 \mathrm{~mm}$. Figure 5 shows the transverse beam cross-plane and in-plane profiles measured at 14 (dmax), 100, and $200 \mathrm{~mm}$ depths with $100 \times 100 \mathrm{~mm}$. Figure 6 shows the percent depth dose curves with SSD of $900 \mathrm{~mm}$ normalized to the depth of $14 \mathrm{~mm}$ in water. Ten fields were measured and depth ranged from 0 to $350 \mathrm{~mm}$. Figure 7 shows the transverse beam cross-plane and in-plane profiles measured at 14 (dmax), 100, and $200 \mathrm{~mm}$ depths with various fields in water. The relative scatter data were measured with various field sizes at the depth of $100 \mathrm{~mm}$ with SSD of $1000 \mathrm{~mm}$ in water. The relative scatter factors ranged from 0.686 at $10 \times 10 \mathrm{~mm}$ to 1.046 at $150 \times 150 \mathrm{~mm}$ (Table 5). 


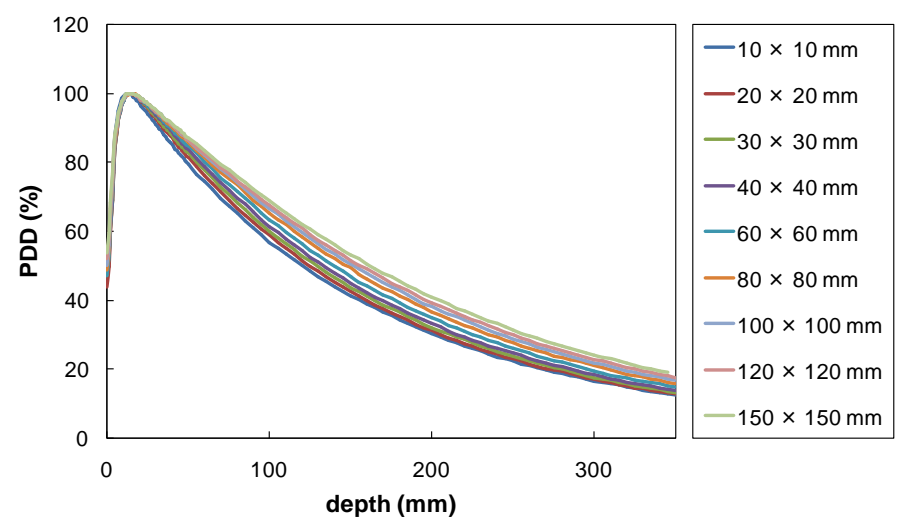

Figure 1. The percent depth dose curves with SSD of $1000 \mathrm{~mm}$ for the PB algorithm at nine field settings: $10 \times 10,20 \times 20,30 \times 30,40 \times 40,60 \times 60,80 \times 80,100 \times 100,120 \times 120$, and $150 \times 150 \mathrm{~mm}$.

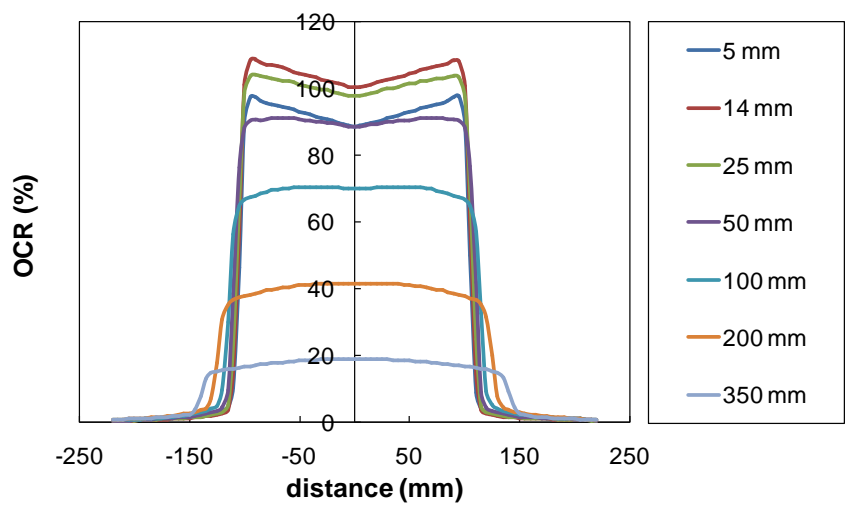

Figure 2. The beam profiles with SSD of $1000 \mathrm{~mm}$ for the PB algorithm with MLC size of $150 \times 150 \mathrm{~mm}$ at the diagonal direction and different depths: 5, 14, 25, 50, 100, 200, and 350 $\mathrm{mm}$.

(a) crossplane

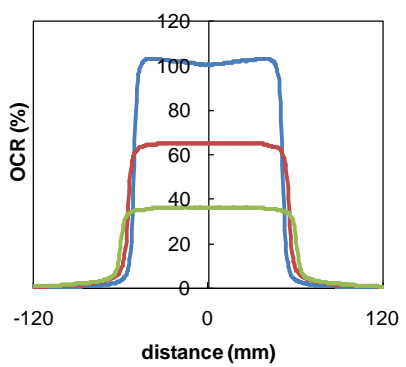

(b) in-plane

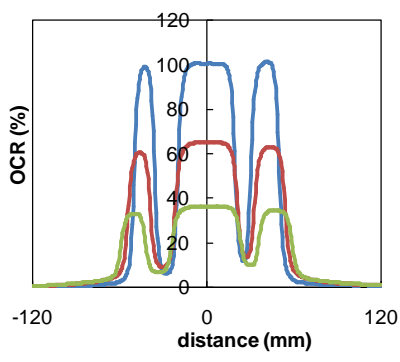

(c) predefined MLC pattern

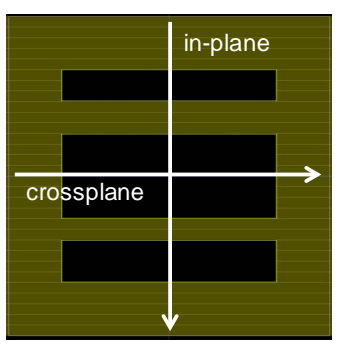

$$
-14 \mathrm{~mm} \quad-100 \mathrm{~mm} \quad-200 \mathrm{~mm}
$$

Figure 3. (a) The cross-plane and (b) in-plane transverse beam profiles with SSD of $100 \mathrm{~mm}$ at depths of 14 (dmax), 100, and $200 \mathrm{~mm}$. (c) predifined MLC pattern.

\subsection{Measurement for MC Algorithm in Air}

Figure 8 shows the CAX profile ranged from 850 to $1150 \mathrm{~mm}$. Figure 9 shows the transverse beam cross-plane and in-plane profiles measured at 850, 1000, and $1150 \mathrm{~mm}$ depths with various fields in air. The relative scatter data were measured with various field sizes with source-chamber distance (SCD) of 1000mm in air. The relative scatter factors ranged from 0.974 at $20 \times 20 \mathrm{~mm}$ to 1.001 at $150 \times 150 \mathrm{~mm}$ (Table 6). 
Table 4. Relative scatter factor with SSD of $1000 \mathrm{~mm}$ at $100-\mathrm{mm}$ depth in water.

\begin{tabular}{cc}
\hline Field size (mm) & Relative scatter factor \\
\hline $10 \times 10$ & 0.697 \\
$20 \times 20$ & 0.820 \\
$30 \times 30$ & 0.866 \\
$40 \times 40$ & 0.896 \\
$60 \times 60$ & 0.941 \\
$80 \times 80$ & 0.974 \\
$100 \times 100$ & 1.000 \\
$120 \times 120$ & 1.022 \\
$150 \times 150$ & 1.046 \\
\hline
\end{tabular}

Table 5. Relative scatter factor with SSD of $900 \mathrm{~mm}$ at $100 \mathrm{~mm}$ depth in water.

\begin{tabular}{cc}
\hline Field size $(\mathrm{mm})$ & Relative scatter factor \\
\hline $10 \times 10$ & 0.686 \\
$30 \times 30$ & 0.868 \\
$50 \times 50$ & 0.921 \\
$70 \times 70$ & 0.958 \\
$100 \times 100$ & 1.000 \\
$150 \times 150$ & 1.046 \\
$50 \times 150$ & 0.964 \\
$100 \times 150$ & 1.023 \\
$150 \times 50$ & 0.961 \\
$150 \times 100$ & 1.019 \\
\hline
\end{tabular}

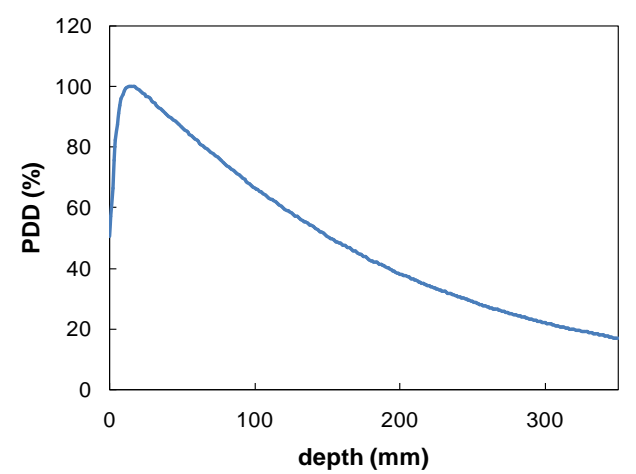

Figure 4. The PDD curves with SSD of $1000 \mathrm{~mm}$ for the MC algorithm at $100 \times 100 \mathrm{~mm}$ in water.

\section{Discussion}

Most reports on the Vero4DRT system describe characteristics of the dynamic tracking system [8] [9]. For typical dosimetric characterization, Nakamura et al. investigated the field characteristics and leaf position accuracy 

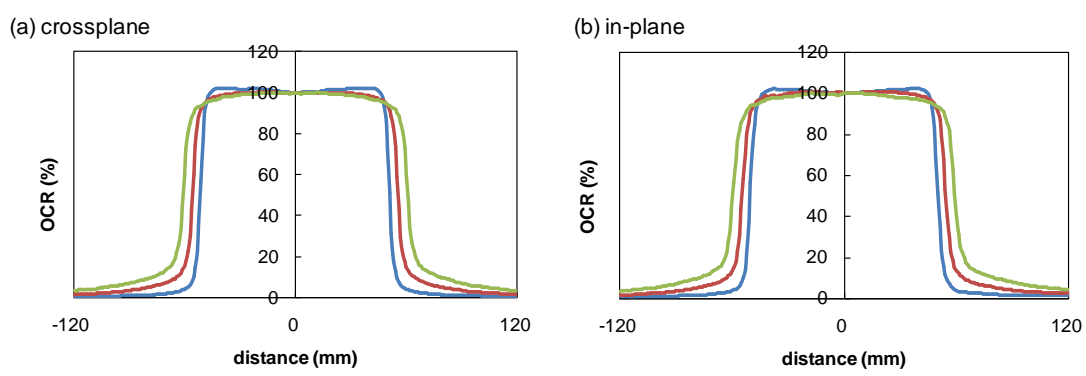

$$
-14 \mathrm{~mm} \quad-100 \mathrm{~mm} \quad-200 \mathrm{~mm}
$$

Figure 5. (a) The cross-plane and (b) in-plane beam profiles with SSD of $1000 \mathrm{~mm}$ for the MC algorithm at 100 $\times 100 \mathrm{~mm}$ in water.

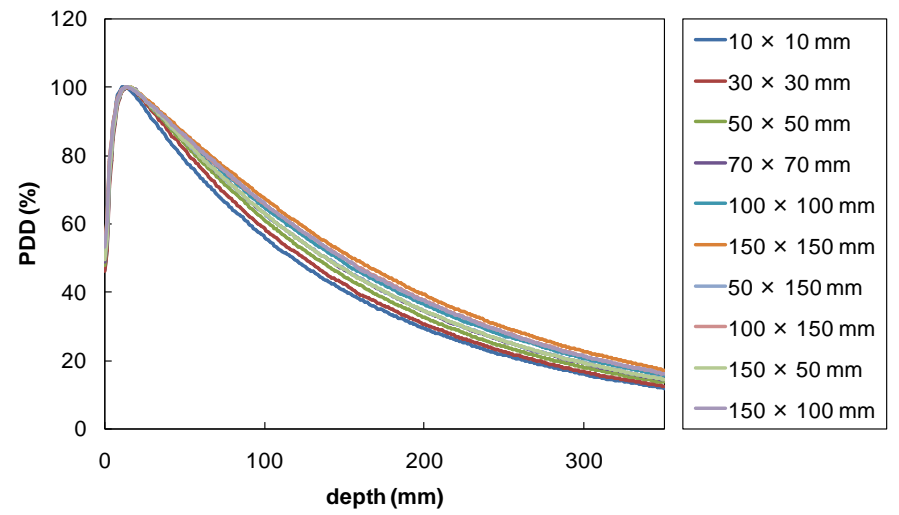

Figure 6. The percent depth dose curves with SSD of $900 \mathrm{~mm}$ for the MC algorithm at 10 field settings in water: $10 \times 10,30 \times 30,50 \times 50,70 \times 70,100 \times 100,150 \times 150,50 \times 150,100 \times 150,150 \times 50$, and $150 \times 100 \mathrm{~mm}$.

(a) crossplane
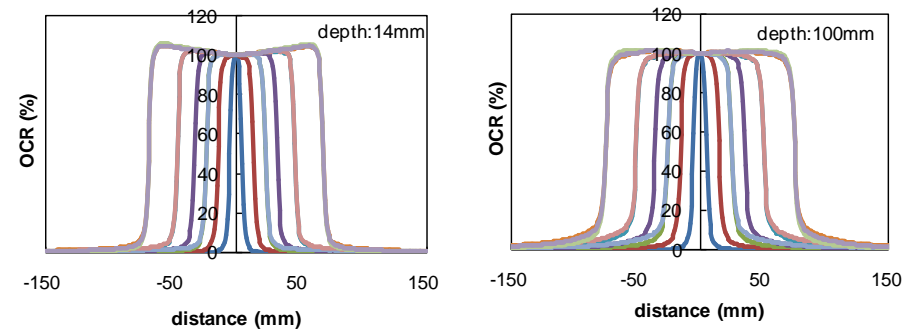

(b) in-plane
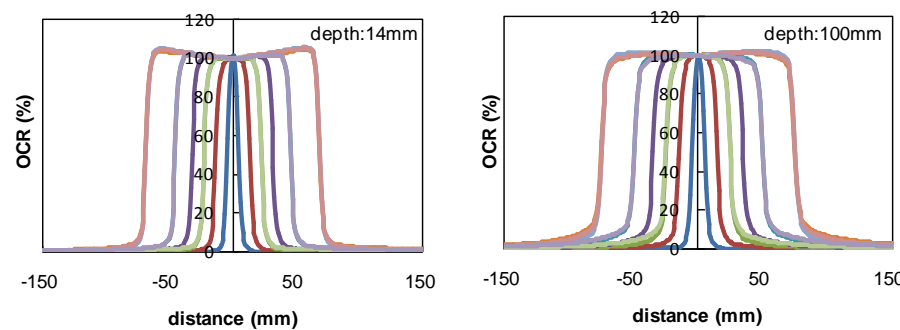

$-150$

distance $(\mathrm{mm})$

$-50$

50
$(\mathrm{~mm})$

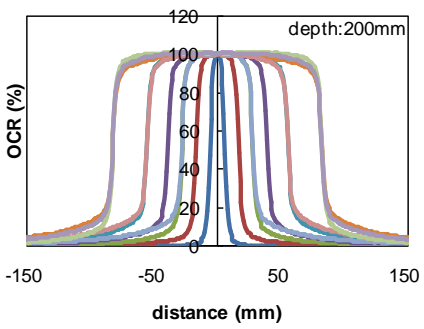

distance $(\mathrm{mm})$

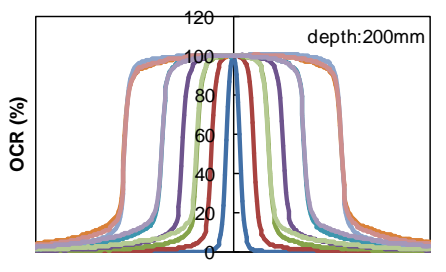

$-150 \quad-50$

distance $(\mathrm{mm})$

\begin{tabular}{|lllll|}
\hline$-10 \times 10 \mathrm{~mm}$ & $-30 \times 30 \mathrm{~mm}$ & $-50 \times 50 \mathrm{~mm}$ & $-70 \times 70 \mathrm{~mm}$ & $-100 \times 100 \mathrm{~mm}$ \\
$-150 \times 150 \mathrm{~mm}$ & $-50 \times 150 \mathrm{~mm}$ & $-100 \times 150 \mathrm{~mm}$ & $-150 \times 50 \mathrm{~mm}$ & $-150 \times 100 \mathrm{~mm}$ \\
\hline
\end{tabular}

Figure 7. (a) The cross-plane and (b) in-plane transverse beam profiles at depths of 14 (dmax), 100, and $200 \mathrm{~mm}$ for the MC algorithm at ten field settings in water: $10 \times 10,30 \times 30,50 \times 50,70 \times 70,100 \times 100,150 \times 150,50 \times$ $150,100 \times 150,150 \times 50$, and $150 \times 100 \mathrm{~mm}$. 


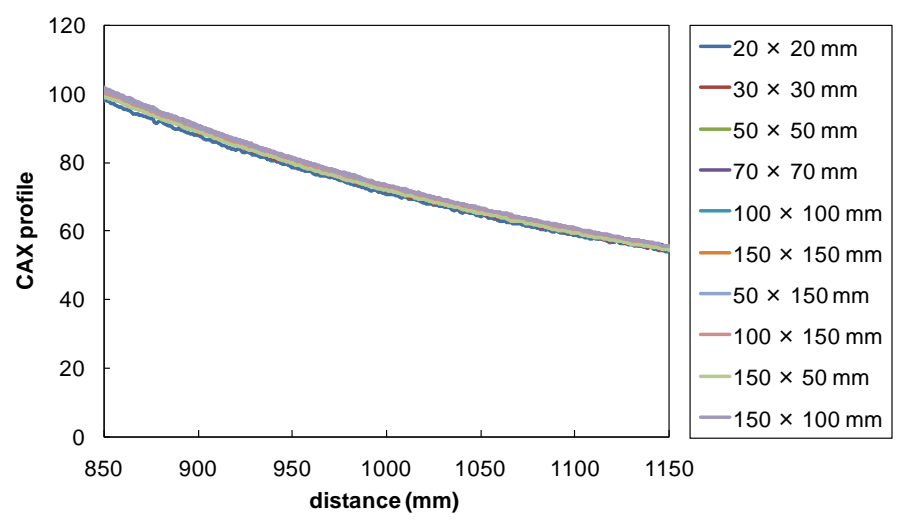

Figure 8. The CAX profile for the MC algorithm at 10 field settings in air: $20 \times 20,30 \times 30,50 \times 50,70 \times 70$, $100 \times 100,150 \times 150,50 \times 150,100 \times 150,150 \times 50$, and $150 \times 100 \mathrm{~mm}$.

(a) crossplane
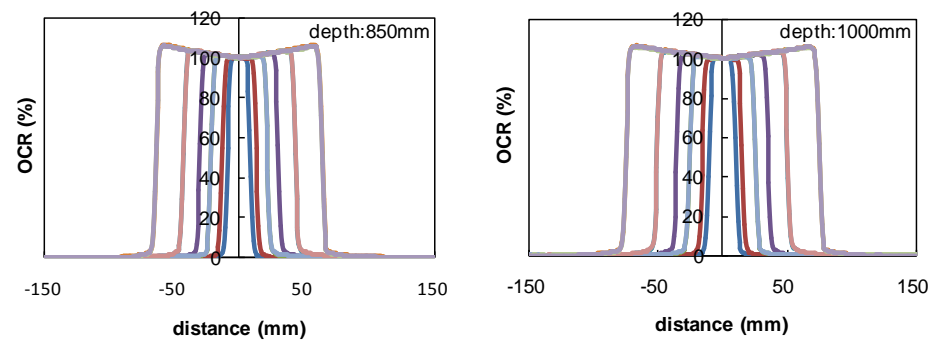

(b) in-plane
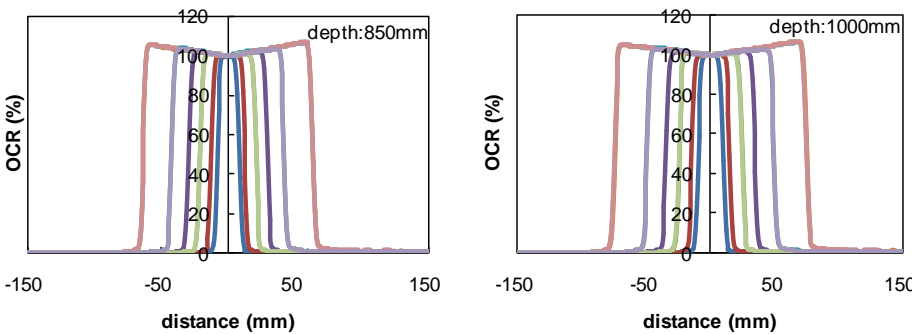

$-50$

distance $(\mathrm{mm})$

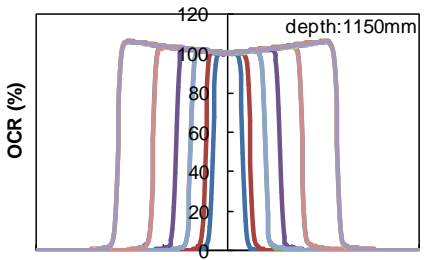

$-150$

$-50$

distance $(\mathrm{mm})$

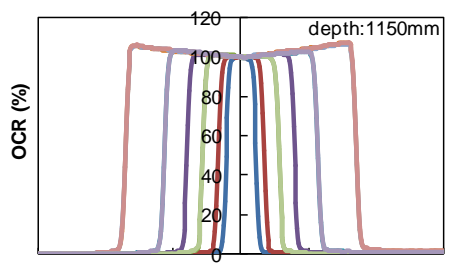

$-150 \quad-50$

distance $(\mathrm{mm})$

$-100 \times 100 \mathrm{~mm}$

Figure 9. (a) The cross-plane and (b) in-plane transverse beam profiles at distance of 850, 1000 and $1150 \mathrm{~mm}$ for the MC algorithm at ten field settings in air: $20 \times 20,30 \times 30,50 \times 50,70 \times 70,100 \times 100,150 \times 150,50 \times 150$, $100 \times 150,150 \times 50$, and $150 \times 100 \mathrm{~mm}$.

of the MLC for MHI-TM2000 using a well-commissioned 6-MV photon beam, EDR2 films, and water-equivalent phantoms [5]. Miura et al. reported the dose linearity and profile flatness/symmetry under low-MU settings of Vero4DRT under low-MU settings [10]. Because the Novalis system likewise supports only iPlan RT, dosimetric characteristics of the Novalis may serve as a reference [11].

The detectors should be selected carefully to improve the accuracy of the dose calculation. Field profile is affected by chamber volume and indicates that a small volume detector is preferred. The detector should be mounted perpendicular to the gun-target direction such that there is minimum volume in the scan direction. Note that the effective measurement point for the PTW31016 pinpoint chamber is $2.4 \mathrm{~mm}$ from the detector top when the detector is positioned in the vertical direction. We also compared relative scatter factor data measured using an EDGE detector (Sun Nuclear Corporation, Melbourne, FL) (data not shown). All measured data in water and air are not used directly during dose calculation in iPlan RT. The commissioning data were sent to BrainLAB and the parameters of the Linac head model were adjusted correspondingly. Check measured data is considered 
Table 6. Relative scatter factor with SCD of $1000 \mathrm{~mm}$ in air.

\begin{tabular}{cc}
\hline Field size $(\mathrm{mm})$ & Relative scatter factor \\
\hline $20 \times 20$ & 0.974 \\
$30 \times 30$ & 0.988 \\
$50 \times 50$ & 0.995 \\
$70 \times 70$ & 0.997 \\
$100 \times 100$ & 1.000 \\
$150 \times 150$ & 1.001 \\
$50 \times 150$ & 0.997 \\
$100 \times 150$ & 1.002 \\
$150 \times 50$ & 1.000 \\
$150 \times 100$ & 1.001 \\
\hline
\end{tabular}

to be more important than measuring beam data. The user must validate the correctness before performing any patient treatment. The incidence of troubles with measured data might be decreased by referral to this study. However, a limitation of this study is that the results are obtained from only one institution. Comparative studies from multiple institutions are needed to verify whether there is a difference with each machine. All the measured PDD and OCR data matched well across the three TrueBeam machines [2].

\section{Conclusion}

We presented a useful measurement dataset for users of the Vero4DRT system. This dataset may help other institutions embarking on Vero4DRT commissioning.

\section{References}

[1] Das, I.J., Cheng, C.W., Watts, R.J., et al. (2008) Accelerator Beam Data Commissioning Equipment and Procedures: Report of the TG-106 of the Therapy Physics Committee of the AAPM. Medical Physics, 35, 4186-4215. http://dx.doi.org/10.1118/1.2969070

[2] Chang, Z., Wu, Q., Adamson, J., et al. (2012) Commissioning and Dosimetric Characteristics of TrueBeam System: Composite Data of Three TrueBeam Machines. Medical Physics, 39, 6981-7018. http://dx.doi.org/10.1118/1.4762682

[3] Chang, Z., Wang, Z., Wu, Q.J., et al. (2008) Dosimetric Characteristics of Novalis Tx System with High Definition Multileaf Collimator. Medical Physics, 35, 4460-4463. http://dx.doi.org/10.1118/1.2977668

[4] Kamino, Y., Takayama, K., Kokubo, M., et al. (2006) Development of a Four-Dimensional Image-Guided Radiotherapy System with a Gimbaled X-Ray Head. International Journal of Radiation Oncology*Biology*Physics, 66, 271278.

[5] Nakamura, M., Sawada, A., Ishihara, Y., et al. (2010) Dosimetric Characterization of a Multileaf Collimator for a New Four-Dimensional Image-Guided Radiotherapy System with a Gimbaled X-Ray Head, MHI-TM2000. Medical Physics, 37, 4684-4691. http://dx.doi.org/10.1118/1.3480510

[6] Ishihara, Y., Sawada, A., Nakamura M., et al. (2014) Development of a Dose Verification System for Vero4DRT Using Monte Carlo Method. Journal of Applied Clinical Medical Physics, 15, 4961.

[7] Braiblab Physics, Technical Reference Guide Revision 1.7, BrainLAB 2013.

[8] Mukumoto, N., Nakamura, M., Sawada, A., et al. (2013) Accuracy Verification of Infrared Marker-Based Dynamic Tumor-Tracking Irradiation Using the Gimbaled X-Ray Head of the Vero4DRT (MHI-TM2000). Medical Physics, 40, Article ID: 041706. http://dx.doi.org/10.1118/1.4794506

[9] Depuydt, T., Verellen, D., Haas, O., et al. (2011) Geometric Accuracy of a Novel Gimbals Based Radiation Therapy Tumor Tracking System. Radiotherapy and Oncology, 98, 365-372. http://dx.doi.org/10.1016/j.radonc.2011.01.015

[10] Miura, H., Osaza, S., Tsuda, S., et al. (2015) Beam Characteristics at Low Dose Monitor Unit Settings for Vero4DRT. International Journal of Medical Physics, Clinical Engineering and Radiation Oncology, 4, 284-289.

[11] Yin, F.F., Zhu, J., Yan, H., et al. (2002) Dosimetric Characteristics of Novalis Shaped Beam Surgery Unit. Medical Physics, 29, 1729-1738. http://dx.doi.org/10.1118/1.1494830 